\title{
Comportamento de reagentes inibidores de incrustação aplicados na perfuração de poços de petróleo
}

\section{Behavior of mineral scale inhibitors applied in drilling of petroleum wells}

\section{Calin Moura da Rosa ${ }^{1}$, Rodrigo Klippel Menger ${ }^{2}$,} Maylline Tavares Gomes ${ }^{2}$, Cristiane Oliveira ${ }^{1}$

\footnotetext{
${ }^{1}$ Mestrado em Avaliação de Impactos Ambientais, Centro Universitário LaSalle - Unilasalle/Canoas, Canoas/RS. Email: olicristiane@gmail.com

${ }^{2}$ Dorf Ketal $^{\mathrm{TM}}$, Rua Luiz Manoel Gonzaga 450 / 601, Porto Alegre/ RS, CEP: 90470-280.
}

\begin{abstract}
RESUMO
A deposição ou incrustação mineral é proveniente do acúmulo de sais inorgânicos que causam problemas operacionais às tubulações e equipamentos aplicados em diversos setores industriais,. Na exploração de petróleo e gás, as incrustações são formadas pela incompatibilidade entre as composições químicas das águas de formação e de injeção e/ou pelas mudanças termodinâmicas do sistema durante estas atividades. A prevenção das incrustações pode ser realizada com a adição substâncias químicas denominadas inibidores de incrustação. Neste trabalho, dois inibidores contendo os grupos funcionais fosfonato e amina (IN-A) e poliméricos (IN-B) foram sintetizados e investigados para aplicação na extração de petróleo em plataformas brasileiras offshore. Os estudos envolveram a avaliação da compatibilidade dos inibidores (IN-A e IN-B) com salmouras e as suas eficiências de inibição de incrustação relacionadas aos íons cálcio. Em todos os estudos, as salmouras foram preparadas com os sais $\mathrm{NaCl}(76,50 \mathrm{~g} / \mathrm{L}), \mathrm{CaCl}_{2} \cdot 2 \mathrm{H}_{2} \mathrm{O}(92,21 \mathrm{~g} / \mathrm{L}), \mathrm{MgCl}_{2} \cdot 6 \mathrm{H}_{2} \mathrm{O}$ (14,42 g/L), $\mathrm{BaCl}_{2} \cdot 2 \mathrm{H}_{2} \mathrm{O}$ (0,04 g/L), $\mathrm{SrCl}_{2} \cdot 6 \mathrm{H}_{2} \mathrm{O}$ (6,33 g/L), KCl (11,59 g/L), $\mathrm{Na}_{2} \mathrm{SO}_{4}(0,68 \mathrm{~g} / \mathrm{L}), \mathrm{NaHCO}_{3}$ $(1,18 \mathrm{~g} / \mathrm{L})$ e $\mathrm{NaCl}(76,50 \mathrm{~g} / \mathrm{L})$, visando simular condições operacionais específicas de plataformas brasileiras. A compatibilidade foi avaliada por análises de turbidez e a eficiência por análises de íons cálcio, posteriormente, expressa em termos percentuais. O IN-A apresentou melhores resultados de compatibilidade, mostrando valores de turbidez menores que 10 NTU para todas as concentrações avaliadas. A eficiência do IN-A foi de, aproximadamente, $80 \%$, indicando um adequado mecanismo quelante de inibição com possível liberação dos complexos solúveis, conforme esperado. Em contraste, o IN-B apresentou menor compatibilidade com as salmouras nas concentrações de 5, 10 e 30 g/L, onde a turbidez foi de 12,7; 15,9 e 31,0 NTU, respectivamente. Além disso, houve queda de eficiência de inibição no decorrer do tempo para as concentrações mais elevadas, de modo que com $30 \mathrm{~g} / \mathrm{L}$ a eficiência foi de 75,78\%, possivelmente, devido a dificuldade de solubilização do polímero com consequente encobrimento dos sítios ativos dos cristais. Os resultados obtidos possibilitaram comparar e avaliar o comportamento dos dois inibidores sintetizados, evidenciando que condições específicas de concentração de sais e perfis de solubilidade são parâmetros muito influentes na formulação e seleção de inibidores de incrustação.
\end{abstract}

Palavras-chave: Inibidores de incrustação. Soluções supersaturadas. Extração de petróleo.

\section{ABSTRACT}

The mineral scale or mineral fouling is from accumulating of inorganic salts, which cause problems to pipes and equipments applied in several industrial sectors. In the gas and oil exploration the mineral scale are formed by incompatibility between the chemical compositions of formation and injection waters and/or thermodynamic changes in the system during these activities. The prevention of scaling may be carrying out with the addition of chemical substances named scale inhibitors. In this work, two inhibitors containing functional groups of phosphonate and amine (IN-A) and polymerics (IN-B) were synthesized and investigated to application in the oil extraction in offshore Brazilian platforms. The studies involved the evaluation of com- 
patibility of the inhibitors (IN-A and IN-B) with brines and their efficiencies to inhibit mineral scale related to calcium ions. In all the studies the brines were prepared with $\mathrm{NaCl}(76.50 \mathrm{~g} / \mathrm{L}), \mathrm{CaCl}_{2 .} 2 \mathrm{H}_{2} \mathrm{O}(92.21 \mathrm{~g} / \mathrm{L})$, $\mathrm{MgCl}_{2} \cdot 6 \mathrm{H}_{2} \mathrm{O}(14.42 \mathrm{~g} / \mathrm{L}), \mathrm{BaCl}_{2} \cdot 2 \mathrm{H}_{2} \mathrm{O}(0.04 \mathrm{~g} / \mathrm{L}), \mathrm{SrCl}_{2} \cdot 6 \mathrm{H}_{2} \mathrm{O}(6.33 \mathrm{~g} / \mathrm{L}), \mathrm{KCl}(11.59 \mathrm{~g} / \mathrm{L}), \mathrm{Na}_{2} \mathrm{SO}_{4}$ $(0.68 \mathrm{~g} / \mathrm{L}), \mathrm{NaHCO}_{3}(1.18 \mathrm{~g} / \mathrm{L})$ e $\mathrm{NaCl}(76.50 \mathrm{~g} / \mathrm{L})$ salts, aiming simulate the specific operational conditions in Brazilian platforms. The compatibility was evaluated by turbidity analyses and the efficiency by calcium ions analyses, later expressed in percentage terms. The IN-A showed better results of compatibility, showing turbidity values less than 10 NTU in all concentrations evaluated. The efficiency of the IN-A was also of, approximately, $80 \%$, indicating a suitable chelating mechanism of inhibition with possible release of soluble complexes, according as expected. In contrast, the IN-B showed smaller compatibility with brines in the 5, 10 e $30 \mathrm{~g} / \mathrm{L}$ concentrations, where the turbidity was of $12.7 ; 15.9$ e $31.0 \mathrm{NTU}$, respectively. In addition, there was decrease in the efficiency of inhibition along the time in the higher concentrations so that in $30 \mathrm{~g} / \mathrm{L}$ the efficiency was of $75.78 \%$ possibly due to difficulties in the solubility of the polymer and therefore cover-up of the active sites of the crystals. The results obtained allowed compare and evaluate the behavior of the two inhibitors synthesized, evidencing that the specific conditions of salt concentrations and solubility patterns are parameters very influents in the formulation and selection of the scale inhibitors.

Keywords: Scale inhibitors. Supersaturated solutions. Oil extraction.

\section{INTRODUÇÃO}

A formação de incrustações de sais inorgânicos sobre tubulações e equipamentos é um problema que geralmente ocorre nos sistemas de injeção de água em poços de extração de petróleo, sendo as incrustações mais comuns formadas por sulfatos de bário, sulfato de cálcio, sulfato de estrôncio e carbonato de cálcio. Em geral, os sulfatos são precipitados devido à mistura da água de injeção (rica em ânions sulfato) com a água de formação (rica em cátions bário, cálcio e estrôncio). Já os carbonatos, normalmente, são precipitados devido às mudanças de temperatura, pressão e/ou pH no sistema [1, 2, 3, 4].

Diante dos desafios relacionados à formação de incrustações, a indústria petroquímica vem buscando o desenvolvimento de produtos, chamados inibidores de incrustação, com o intuito de prevenir a formação dos principais sais presentes nas águas de exploração do petróleo. Atualmente, o uso destes reagentes pode ainda ser considerado dispendioso, entretanto, levando-se em consideração todos os custos e as perdas de produção oriundas da incrustação, é possível observar uma importante vantagem econômica envolvida. Além disso, acredita-se que os novos produtos inibidores possam oferecer mais possibilidades de tratamento das águas de diferentes composições e maior viabilidade econômica, sendo ainda relevante o desenvolvimento de pesquisas nesta área[ㅌ, 6,7].

Os inibidores de incrustação podem pertencer às diversas classes químicas e apresentar diferentes mecanismos de inibição, podendo atuar, principalmente, por quelação ou modificação dos cristais. Um inibidor com propriedades quelantes realiza uma ligação de complexação com o cátion que forma a incrustação e, deste modo, o cátion permanece complexado no inibidor em solução e, portanto, não disponível para a formar uma incrustação. Um exemplo deste tipo de produto são os inibidores a base de EDTA (ácido etileno diamino tetracético) que complexam íons cálcio e magnésio [8]]. Já na modificação do crescimento de cristais, o inibidor forma uma ligação no sítio ativo de crescimento dos cristais, impedindo o crescimento ordenado e alterando a forma dos cristais que, assim, tendem a não se aderir sobre as superfícies, permanecendo dispersos na fase líquida. Alguns produtos orgânicos naturais, tais como ligninas e taninos, são usados com esta finalidade, sendo, recentemente, o uso de polímeros e copolímeros sintéticos (poliacrilatos, maleicos, fosfinocarboxílicos, entre outros) mais aplicado. Em alguns casos, um único reagente pode apresentar duas ou mais características mencionadas, sendo que a escolha deve levar em consideração os principais íons presentes nas águas, a tendência de acúmulo de material orgânico, o tipo de incrustação, as condições de temperatura, pressão e pH e o custo global da aplicação [ $\underline{9,10,11,12,13}$.

Assim, nesta linha de pesquisa o maior desafio é, provavelmente, avaliar a performance de inibição nas condições operacionais de exploração, havendo uma ampla variação nos parâmetros influentes no processo. Os principais testes realizados para avaliar um inibidor de incrustação são os de compatibilidade e de eficiência. Nos testes de compatibilidade, o inibidor é misturado com soluções salinas (salmouras) em composições e temperaturas que simulam as condições operacionais da extração com subsequente avaliação das possíveis interações entre os íons em solução e o inibidor, bem como, a solubilidade do mesmo. Nestes testes, a turbidez é o parâmetro de resposta, sendo estabelecidos valores para classificar os inibidores como compatíveis ou incompatíveis. Já o teste de eficiência visa avaliar a capacidade do reagente para inibir a formação da incrustação, podendo ser realizado por testes dinâmicos ou estáticos. Os testes dinâmicos, denominados Scale Loop Test [9]], utilizam instrumentos para simular condições de altas pressões e temperaturas nas explorações. 
Já os testes estáticos, utilizam métodos analíticos para quantificar o principal íon gerador da incrustação, sendo a análise de íons cálcio de extrema importância para a investigação de um inibidor para uso em plataformas brasileiras, tendo em vista que na camada pré-sal as rochas dos reservatórios são de origem carbonática $[\underline{9,14}]$.

Dentro deste contexto, o presente trabalho visa sintetizar e analisar dois tipos de inibidores de incrustação com o propósito de avaliar suas eficiências de inibição de incrustação formada por íons cálcio, bem como, suas compatibilidades com salmouras catiônica e aniônica que simulam as composições das águas envolvidas na exploração de uma plataforma de petróleo brasileira.

\section{MATERIAIS E MÉTODOS}

\subsection{Materiais e reagentes}

Os inibidores estudados foram desenvolvidos em parceria com a empresa DorfKetal ${ }^{\mathrm{TM}}$, sendo classificados como IN-A (contendo grupos funcionais fosfonato e amina) e IN-B (contendo grupos poliméricos). As formulações destes reagentes visaram produtos com diferentes mecanismos de inibição, possuindo o IN-A ação quelante e o IN-B ação modificadora do crescimento do cristal. Por questões de sigilo industrial, a composição dos reagentes não poderá ser descrita nesta seção.

As soluções aquosas salinas, denominadas salmouras, foram preparadas com os reagentes cloreto de sódio P.A., cloreto de cálcio dihidratado P.A., cloreto de magnésio hexahidratado, cloreto de bário dihidratado P.A., cloreto de estrôncio hexahidratado, cloreto de potássio P.A., sulfato de sódio P.A. e bicarbonato de sódio P.A., visando simular as condições operacionais das águas na exploração em uma plataforma brasileira de petróleo. Adicionalmente, hidróxido de potássio P.A. foi aplicado para ajuste de $\mathrm{pH}$ das salmouras nos estudos.

Adicionalmente, os reagentes cloreto de amônio P.A., hidróxido de amônio P.A., EDTA P.A., cloreto de magnésio P.A., negro de ericromo-T P.A., carbonato de cálcio, trietanolamina, ácido clorídrico P.A., hidróxido de alumínio P.A.foram usados para medir a concentração dos íons cálcio para determinação das eficiências dos reagentes.

\subsection{Metodologia}

As soluções de inibidores foram formuladas uma semana antes da realização dos estudos, sendo armazenadas em frascos de vidro transparente. As concentrações dos inibidores investigadas foram 0,5, 1, 5, 10 e $30 \mathrm{~g} / \mathrm{L}$, sendo estes valores escolhidos com base nas condições do processo informadas pela empresa petrolífera exploradora.

No momento dos estudos foram preparadas as soluções aquosas salinas (salmouras), sendo uma com cátions $\left(\mathrm{Na}^{+}, \mathrm{Ca}^{2+}, \mathrm{Mg}^{2+}, \mathrm{Ba}^{2+}, \mathrm{Sr}^{2+}\right.$ e $\left.\mathrm{K}^{+}\right)$para simular as águas de formação e outra com ânions $\left(\mathrm{SO}_{4}{ }^{2-}\right.$, $\mathrm{HCO}_{3}{ }^{-}$e $\mathrm{Cl}^{-}$) para simular as águas de injeção que estão presentes nas operações. A Tabela 1 mostra as concentrações utilizadas nestas soluções.

Tabela 1: Composição das salmouras catiônica e aniônica utilizadas nos estudos.

\begin{tabular}{ccc} 
SALMOURA & COMPOSIÇÃO & CONCENTRAÇÃO, g/L \\
\hline & $\mathrm{NaCl}$ & 76,50 \\
CATIÔNICA: ÁGUA & $\mathrm{CaCl}_{2} \cdot 2 \mathrm{H}_{2} \mathrm{O}$ & 92,21 \\
DE FORMAÇÃo & $\mathrm{MgCl}_{2} \cdot 6 \mathrm{H}_{2} \mathrm{O}$ & 14,42 \\
& $\mathrm{BaCl}_{2} \cdot 2 \mathrm{H}_{2} \mathrm{O}$ & 0,04 \\
& $\mathrm{SrCl}_{2} \cdot 6 \mathrm{H}_{2} \mathrm{O}$ & 6,33 \\
& $\mathrm{KCl}_{2}$ & 11,59 \\
\hline \multirow{2}{*}{ ANIÔNICA: ÁGUA } & $\mathrm{Na}_{2} \mathrm{SO}_{4}$ & 0,68 \\
DE INJEÇÃO & $\mathrm{NaHCO}_{3}$ & 1,18 \\
& $\mathrm{NaCl}^{*}$ & 76,50 \\
\hline
\end{tabular}

A compatibilidade e a eficiência dos inibidores foram investigadas segundo as metodologias descritas 
por [16] e [15]. A compatibilidade está relacionada às capacidades de manter os cátions formadores de incrustação em solução, bem como, de solubilização dos inibidores. Já a eficiência está relacionada com a capacidade dos inibidores em evitar a formação de sais de cálcio (incrustação).

O procedimento experimental iniciou com o preparo das salmouras em água deionizada e em concentrações específicas para simular as condições do processo de exploração, conforme descrito na Tabela 1. Volumes (50 mL) de cada salmoura (catiônica e aniônica) foram medidos em balões volumétricos e transferidos para frascos de vidro de $100 \mathrm{~mL}$ de capacidade. Após, foram adicionadas gotas de uma solução de $\mathrm{KOH}$ (10\%), sob agitação com bastão de vidro e eletrodo imerso, visando ajustar o pH em 6,0 para simular as condições de exploração. Após o alcance da estabilidade do pH, as concentrações de 500, 1000, 5000, 10000 e $30 \mathrm{~g} / \mathrm{L}$ dos inibidores (IN-A e IN-B) foram adicionadas aos frascos que foram fechados e agitados manualmente por 5 min para homogeneização das misturas. Em seguida, os frascos foram inseridos em um banhomaria a temperatura de $90{ }^{\circ} \mathrm{C}$, simulando a temperatura do processo de extração.

Os estudos de compatibilidade dos inibidores foram realizados em duplicata utilizando 10 frascos para as condições citadas e 2 frascos de brancos (sem adição dos inibidores). Amostras de cada frasco foram coletadas nos períodos de 0, 1, 2 e 24 h e analisadas quando ao $\mathrm{pH}$ e turbidez pelos métodos instrumentais [17]. As condições experimentais que promoveram valores de turbidez menores que 10 NTU, classificaram os inibidores como compatíveis, conforme metodologia desenvolvida pelo fabricante dos reagentes investigados.

Os estudos de eficiência dos inibidores também foram realizados em duplicata utilizando 10 frascos para as condições citadas, porém quatro frascos de brancos (sem adição dos inibidores), sendo dois sem aquecimento, visando quantificar a concentração de íons cálcio antes da precipitação (sem aquecimento) e após a precipitação (com aquecimento)[ㅌ]. Amostras dos frascos foram coletadas nos períodos de 0, 1, 2 e 24 h e analisadas quando à concentração de íons cálcio, de acordo com [15]. Esta análise de íons cálcio foi realizada pelo método complexométrico, onde EDTA titula uma amostra em pH 10 para determinar íons cálcio e magnésio ou em pH entre 12-13 para determinar apenas íons cálcio (conforme aplicado no presente estudo). Neste procedimento, uma alíquota volumétrica da amostra foi transferida para um erlenmeyer de 150 $\mathrm{mL}$ e o volume foi ajustado para, aproximadamente, $50 \mathrm{~mL}$. Após, $1 \mathrm{~mL}$ de trietanolamina foi adicionado para eliminar interferência de manganês, seguido de $1 \mathrm{~mL}$ de $\mathrm{NaCN}$, de $1 \mathrm{~mL}$ de solução de $\mathrm{NaOH}$ para atingir um valor de $\mathrm{pH}$ entre 12 a 13. Imediatamente após a adição de 0,2 g de indicador negro de ericromo-T a titulação foi realizada com uma solução padronizada de EDTA $(0,01 \mathrm{M})$. Posteriormente, uma amostra de branco também foi titulada. A concentração de íons cálcio foi determinada pela equação 1, onde, $A$ é o volume, em mL, da solução padronizada de EDTA gasta para titular o cálcio na amostra menos o volume, em mL, gasto desta solução para titular o branco, $B$ é a molaridade da solução de EDTA padronizada e $D$ é o volume da amostra.

[íons.cálcio], $m g / L=\left(\frac{A * B}{D}\right) * 40 * 100$

Adicionalmente, o teste de eficiência estática para íons cálcio foi realizado pelo método [16], onde a partir dos resultados da análise de concentração de íons cálcio na amostra depois da precipitação $\left(C_{a}\right)$, no branco depois da precipitação, ou seja, com aquecimento $\left(C_{b}\right)$ e no branco antes da precipitação, ou seja, sem aquecimento $\left(C_{c}\right)$, a eficiência foi determinada em termos de percentuais pela equação 2 . Assim, os valores superiores à 80\% caracterizaram um inibidor de incrustações eficaz, conforme estabelecido por [16].

$$
\text { Eficiência }=\frac{\left(C_{a}-C_{b}\right)}{\left(C_{c}-C_{b}\right)} * 100 \%
$$

\section{RESULTADOS E DISCUSSÃO}

Os resultados obtidos nos estudos de compatibilidade para IN-A e IN-B podem ser visualizados na Tabela 2 a partir dos valores de turbidez e $\mathrm{pH}$. 
Tabela 2: Valores de pH e turbidez das amostras em diferentes concentrações e tempos de contato nos estudos de compatibilidade dos IN-A e IN-B. Condições experimentais: $\mathrm{V}_{\text {salmoura }}: 50 \mathrm{~mL}, \mathrm{pH}_{\text {inicial }}: 6,0, \mathrm{~T}: 90^{\circ} \mathrm{C}$.

\begin{tabular}{|c|c|c|c|c|c|c|c|c|}
\hline PRODUTO & \multicolumn{8}{|c|}{ TEMPO DE REAÇÃO } \\
\hline & \multicolumn{2}{|r|}{$\mathbf{O h}$} & \multicolumn{2}{|c|}{01 h } & \multicolumn{2}{|r|}{02 h } & \multicolumn{2}{|r|}{$24 \mathrm{~h}$} \\
\hline Branco & pH & $\begin{array}{c}\text { Turbidez } \\
\text { (NTU) }\end{array}$ & pH & $\begin{array}{c}\text { Turbidez } \\
\text { (NTU) }\end{array}$ & pH & $\begin{array}{c}\text { Turbidez } \\
\text { (NTU) }\end{array}$ & $\mathbf{p H}$ & \\
\hline & 6,00 & $\begin{array}{c}7,965 \pm \\
0,03\end{array}$ & $\begin{array}{c}6,01 \pm \\
0,01\end{array}$ & $9,34 \pm 1,92$ & $\begin{array}{c}6,065 \pm \\
0,05\end{array}$ & $8,095 \pm 0,15$ & $\begin{array}{c}6,11 \pm \\
0,01\end{array}$ & $8,07 \pm 0,10$ \\
\hline \multirow[b]{2}{*}{ [IN-A], g/L } & \multicolumn{2}{|r|}{$\mathbf{0 ~ h}$} & \multicolumn{2}{|c|}{$01 \mathrm{~h}$} & \multicolumn{2}{|r|}{$02 \mathrm{~h}$} & \multicolumn{2}{|r|}{$24 \mathrm{~h}$} \\
\hline & pH & $\begin{array}{c}\text { Turbidez } \\
\text { (NTU) }\end{array}$ & pH & $\begin{array}{c}\text { Turbidez } \\
\text { (NTU) }\end{array}$ & pH & $\begin{array}{c}\text { Turbidez } \\
\text { (NTU) }\end{array}$ & pH & $\begin{array}{c}\text { Turbidez } \\
\text { (NTU) }\end{array}$ \\
\hline 0,5 & 6,40 & $7,80 \pm 0,02$ & 6,47 & $8,17 \pm 0,02$ & 6,21 & $8,18 \pm 0,02$ & 6,39 & $8,20 \pm 0,01$ \\
\hline 1,0 & 6,40 & $8,12 \pm 0,01$ & 6,75 & $8,15 \pm 0,02$ & 6,95 & $8,21 \pm 0,02$ & 6,68 & $8,18 \pm 0,01$ \\
\hline 5,0 & 6,40 & $8,10 \pm 0,03$ & 6,78 & $8,11 \pm 0,02$ & 7,05 & $8,11 \pm 0,01$ & 7,31 & $8,12 \pm 0,02$ \\
\hline 10,0 & 6,40 & $8,18 \pm 0,01$ & 6,75 & $8,18 \pm 0,01$ & 7,00 & $8,20 \pm 0,01$ & 7,05 & $8,22 \pm 0,02$ \\
\hline 30,0 & 6,40 & $8,19 \pm 0,01$ & 6,35 & $8,21 \pm 0,02$ & 6,54 & $8,21 \pm 0,01$ & 6,14 & $8,21 \pm 0,01$ \\
\hline \multirow[b]{2}{*}{ [IN-B], g/L } & \multicolumn{2}{|r|}{$\mathbf{O h}$} & \multicolumn{2}{|c|}{$01 \mathrm{~h}$} & \multicolumn{2}{|r|}{$02 \mathrm{~h}$} & \multicolumn{2}{|r|}{$24 \mathrm{~h}$} \\
\hline & pH & $\begin{array}{c}\text { Turbidez } \\
\text { (NTU) }\end{array}$ & pH & $\begin{array}{c}\text { Turbidez } \\
\text { (NTU) }\end{array}$ & pH & $\begin{array}{c}\text { Turbidez } \\
\text { (NTU) }\end{array}$ & pH & $\begin{array}{c}\text { Turbidez } \\
\text { (NTU) }\end{array}$ \\
\hline 0,5 & 8,10 & $8,12 \pm 0,02$ & 7,88 & $8,17 \pm 0,03$ & 8,90 & $8,18 \pm 0,02$ & 7,90 & $8,18 \pm 0,01$ \\
\hline 1,0 & 8,10 & $8,15 \pm 0,01$ & 7,87 & $8,18 \pm 0,01$ & 8,06 & $8,21 \pm 0,02$ & 8,14 & $8,25 \pm 0,01$ \\
\hline 5,0 & 8,10 & $9,14 \pm 0,01$ & 7,84 & $22,8 \pm 0,01$ & 8,02 & $22,4 \pm 0,01$ & 8,13 & $12,7 \pm 0,01$ \\
\hline 10,0 & 8,10 & $15,3 \pm 0,01$ & 7,92 & $28,9 \pm 0,01$ & 7,81 & $28,7 \pm 0,01$ & 7,83 & $15,9 \pm 0,02$ \\
\hline 30,0 & 8,10 & $49,3 \pm 0,01$ & 7,86 & $57,1 \pm 0,01$ & 7,85 & $57,7 \pm 0,01$ & 7,88 & $31,0 \pm 0,01$ \\
\hline
\end{tabular}

A partir destes resultados (Tabela 2) foi possível observar que não houve mudança significativa de pH, com a adição do IN-A. No entanto, para o IN-B ocorreu um pequeno aumento deste parâmetro em decorrência de sua formulação.

Com relação à turbidez, o inibidor IN-A apresentou valores mais estáveis e menores que 10,0 NTU. Estes valores indicam uma boa compatibilidade com as salmouras e, portanto, uma boa interação eletrostática e solubilidade do inibidor nas condições investigadas. Ao término destes estudos foi observada uma leve precipitação nos frascos contendo $0,5 \mathrm{~g} / \mathrm{L}$ do inibidor. Este fato, possivelmente, decorreu da baixa concentração do inibidor que foi insuficiente para evitar o completo mecanismo de precipitação dos sais. Adicionalmente, é importante salientar que houve turbidez nas amostras de branco em decorrência da supersaturação das soluções nas concentrações de salmouras preparadas.

Já a compatibilidade do IN-B apareceu satisfatória para baixas concentrações (0,5 e 1,0 g/L), onde as soluções permaneceram com valores de turbidez abaixo de 10 NTU, indicando que, além de compatível, a composição polimérica não apresentou problemas de solubilidade. Em contraste, nas concentrações de 5, 10 e $30 \mathrm{~g} / \mathrm{L}$, a turbidez foi elevada em todos os períodos investigados, possivelmente, devido à dificuldade de solubilização que ocorre com o aumento da concentração das cadeias poliméricas em solução [18].

Adicionalmente, a Tabela 3 mostra os resultados das concentrações dos íons cálcio determinadas para avaliar a eficiência dos reagentes para inibir o mecanismo de incrustação. 
Tabela 3: Concentração de íons cálcio nas soluções investigadas em diferentes concentrações dos inibidores IN-A e IN-B e tempos de contato. Condições experimentais: $\mathrm{V}_{\text {salmoura }}: 50 \mathrm{~mL}, \mathrm{pH}_{\text {inicial }}: 6, \mathrm{~T}: 90^{\circ} \mathrm{C}$ (exceto para o branco sem aquecimento).

\begin{tabular}{|c|c|c|c|c|c|c|c|c|c|}
\hline PRODUTO & \multicolumn{4}{|c|}{ TEMPO DE CONTATO, h } & PRODUTO & \multicolumn{4}{|c|}{ TEMPO DE CONTATO, h } \\
\hline [IN-A], g/L & $\mathbf{0 ~ h}$ & 01h & $02 \mathrm{~h}$ & $24 \mathrm{~h}$ & $\begin{array}{c}{[\mathrm{IN}-\mathrm{B}]} \\
(\mathrm{g} / \mathrm{l})\end{array}$ & $\mathbf{O} \mathbf{h}$ & $01 \mathrm{~h}$ & 02 h & $24 \mathrm{~h}$ \\
\hline & \multicolumn{4}{|c|}{ [ÍONS CÁLCIO], g/L } & & \multicolumn{4}{|c|}{ [ÍONS CÁLCIO], g/L } \\
\hline $\begin{array}{c}0 \text { (sem aque- } \\
\text { cimento) }\end{array}$ & $\begin{array}{c}2,86 \pm \\
0,03 \\
\end{array}$ & $\begin{array}{r}2,86 \\
\pm 0,01 \\
\end{array}$ & $\begin{array}{r}2,86 \\
\pm 0,01 \\
\end{array}$ & $\begin{array}{r}2,86 \\
\pm 0,01 \\
\end{array}$ & $\begin{array}{c}0 \text { (sem aque- } \\
\text { cimento) }\end{array}$ & $\begin{array}{r}2,85 \\
\pm 0,03 \\
\end{array}$ & $\begin{array}{r}2,85 \\
\pm 0,03 \\
\end{array}$ & $\begin{array}{r}2,85 \\
\pm 0,02 \\
\end{array}$ & $\begin{array}{r}2,86 \\
\pm 0,03 \\
\end{array}$ \\
\hline $\begin{array}{c}0 \text { (com aque- } \\
\text { cimento) }\end{array}$ & $\begin{array}{c}2,45 \\
\pm 0,01 \\
\end{array}$ & $\begin{array}{c}2,41 \\
\pm 0,01 \\
\end{array}$ & $\begin{array}{c}2,41 \\
\pm 0,02 \\
\end{array}$ & $\begin{array}{r}2,40 \\
\pm 0,02 \\
\end{array}$ & $\begin{array}{c}0 \text { (com aque- } \\
\text { cimento) }\end{array}$ & $\begin{array}{c}2,43 \\
\pm 0,01 \\
\end{array}$ & $\begin{array}{c}2,43 \\
\pm 0,01 \\
\end{array}$ & $\begin{array}{r}2,43 \\
\pm 0,02 \\
\end{array}$ & $\begin{array}{r}2,43 \\
\pm 0,02 \\
\end{array}$ \\
\hline 0,5 & $\begin{array}{c}2,78 \pm \\
0,05 \\
\end{array}$ & $\begin{array}{c}2,77 \pm \\
0,07 \\
\end{array}$ & $\begin{array}{c}2,75 \pm \\
0,01 \\
\end{array}$ & $\begin{array}{c}2,74 \pm \\
0,02 \\
\end{array}$ & 0,5 & $\begin{array}{c}2,77 \\
\pm 0,07 \\
\end{array}$ & $\begin{array}{c}2,77 \pm \\
0,03 \\
\end{array}$ & $\begin{array}{c}2,77 \pm \\
0,05 \\
\end{array}$ & $\begin{array}{c}2,77 \pm \\
0,03 \\
\end{array}$ \\
\hline 1,0 & $\begin{array}{r}2,78 \\
\pm 0,05\end{array}$ & $\begin{array}{c}2,76 \pm \\
0,01\end{array}$ & $\begin{array}{c}2,78 \pm \\
0,03\end{array}$ & $\begin{array}{c}2,76 \pm \\
0,01\end{array}$ & 1,0 & $\begin{array}{c}2,77 \pm \\
0,03\end{array}$ & $\begin{array}{c}2,77 \pm \\
0,01\end{array}$ & $\begin{array}{c}2,77 \pm \\
0,07\end{array}$ & $\begin{array}{c}2,77 \pm \\
0,03 \\
\end{array}$ \\
\hline 5,0 & $\begin{array}{c}2,78 \pm \\
0,03\end{array}$ & $\begin{array}{c}2,78 \pm \\
0,04\end{array}$ & $\begin{array}{c}2,77 \pm \\
0,01\end{array}$ & $\begin{array}{c}2,78 \pm \\
0,03\end{array}$ & 5,0 & $\begin{array}{c}2,77 \pm \\
0,03\end{array}$ & $\begin{array}{c}2,77 \pm \\
0,01\end{array}$ & $\begin{array}{c}2,77 \pm \\
0,03 \\
\end{array}$ & $\begin{array}{c}2,77 \pm \\
0,09\end{array}$ \\
\hline 10,0 & $\begin{array}{c}2,79 \pm \\
0,05 \\
\end{array}$ & $\begin{array}{c}2,78 \pm \\
0,04 \\
\end{array}$ & $\begin{array}{c}2,78 \pm \\
0,03 \\
\end{array}$ & $\begin{array}{c}2,79 \pm \\
0,03 \\
\end{array}$ & 10,0 & $\begin{array}{c}2,77 \pm \\
0,04 \\
\end{array}$ & $\begin{array}{c}2,76 \pm \\
0,01 \\
\end{array}$ & $\begin{array}{c}2,76 \pm \\
0,03 \\
\end{array}$ & $\begin{array}{c}2,77 \pm \\
0,03 \\
\end{array}$ \\
\hline 30,0 & $\begin{array}{c}2,79 \pm \\
0,08\end{array}$ & $\begin{array}{c}2,79 \pm \\
0,01\end{array}$ & $\begin{array}{c}2,79 \pm \\
0,03\end{array}$ & $\begin{array}{c}2,79 \pm \\
0,03\end{array}$ & 30,0 & $\begin{array}{c}2,75 \pm \\
0,03\end{array}$ & $\begin{array}{c}2,74 \pm \\
0,01\end{array}$ & $\begin{array}{c}2,74 \pm \\
0,01\end{array}$ & $\begin{array}{c}2,75 \pm \\
0,05\end{array}$ \\
\hline
\end{tabular}

A partir destes resultados de concentração de íons cálcio em solução, as eficiências calculadas para IN-A, e IN-B (Figuras 1 e 2) alcançaram, na maioria dos casos, valores próximos à 80\%, sendo este valor o mínimo aceitável para um inibidor ser considerado compatível, conforme descrito por [16].

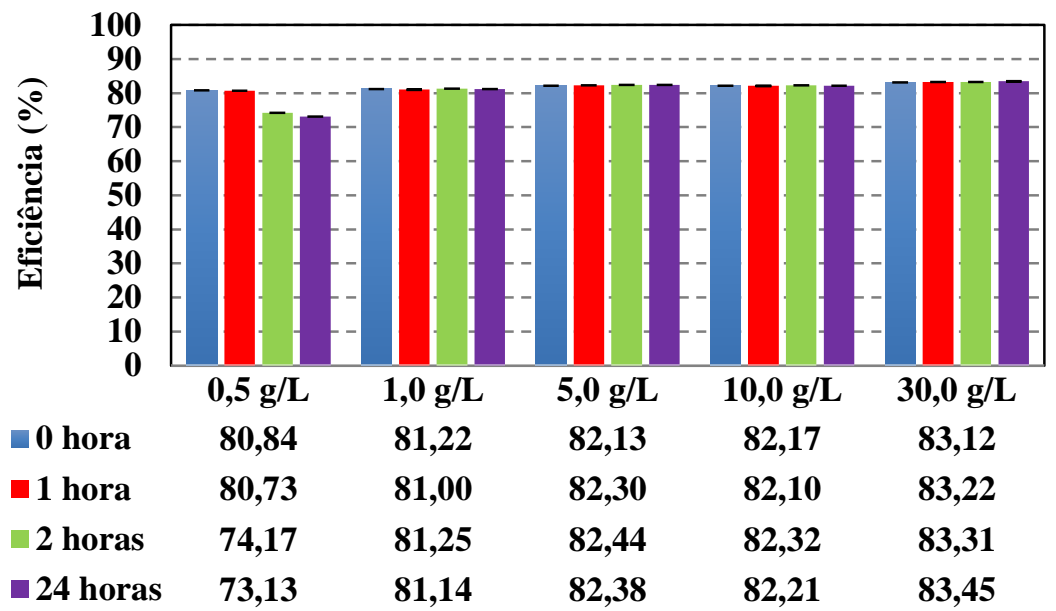

Figura 1: Eficiência do IN-A para inibir a incrustação por íons cálcio em diferentes concentrações e tempos de contato com as salmouras.

Com base nos resultados da Figura 1, é possível observar que a eficiência do IN-A na condição de 0,5 
g/L foi levemente mais baixa, possivelmente devido à menor concentração de inibidor que não foi suficiente para complexar todos os cátions formadores de incrustação. Acima de 1 g/L é possível evidenciar uma eficiência de, aproximadamente, $80 \%$ para todas as condições, indicando que existe uma concentração ótima onde os grupamentos do inibidor realizam a ação quelante no momento de supersaturação da solução, de modo que o fosfonato, possivelmente, atuou complexando os cátions presentes e as aminas, possivelmente, aumentaram a eficiência através dos pares de elétrons livres, sendo esta associação viável para o sequestro de cátions metálicos que permanecem solúveis na fase líquida [8]]. Além disso, a formulação do inibidor mostrou-se totalmente miscível em água, não ocasionando problemas de estabilidade devido à solubilização nas condições investigadas.

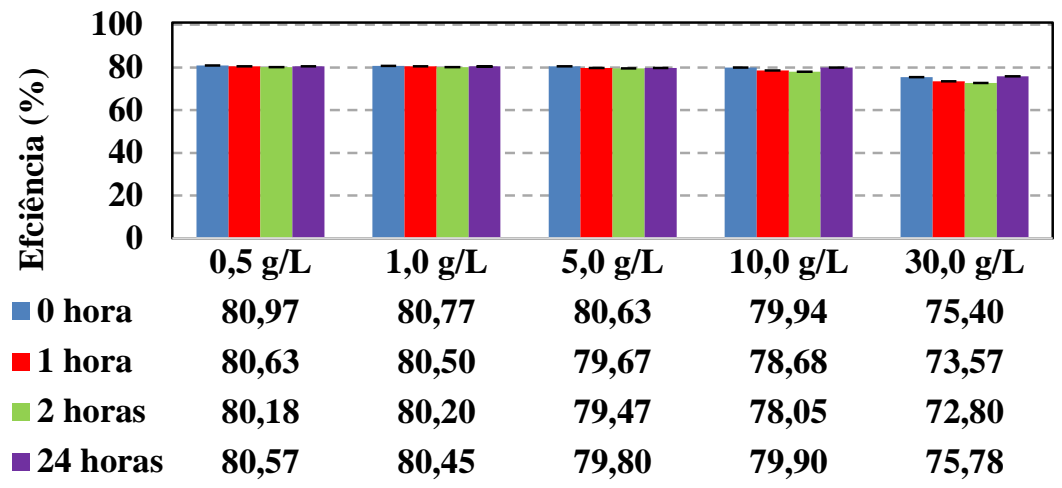

Figura 2: Eficiência do IN-B para inibir a incrustação por íons cálcio em diferentes concentrações e tempos de contato com as salmouras.

As eficiências obtidas com a adição do IN-B (Figura 2) também foram muito próximas de $80 \%$ na maioria das condições investigadas. No caso da concentração de $30 \mathrm{~g} / \mathrm{L}$, os valores ficaram abaixo de $80 \%$, sendo considerado por [16] um inibidor incompatível. Possivelmente, este fato decorreu da redução da solubilidade da estrutura polimérica do IN-B que, consequentemente, inviabilizou uma completa interação do polímero com os sítios ativos dos cristais. De acordo com alguns autores [19, 20], macromoléculas poliméricas interagem com os íons em solução apresentando dificuldades de difusão das cadeias em algumas condições de salinidade.

Complementarmente aos resultados analíticos, foi possível visualizar diferenças expressivas na turbidez das amostras, conforme mostrado nas Figuras 3 e 4, de modo que o IN-B apresentou maior turbidez com a formação de precipitados sedimentados em algumas amostras.

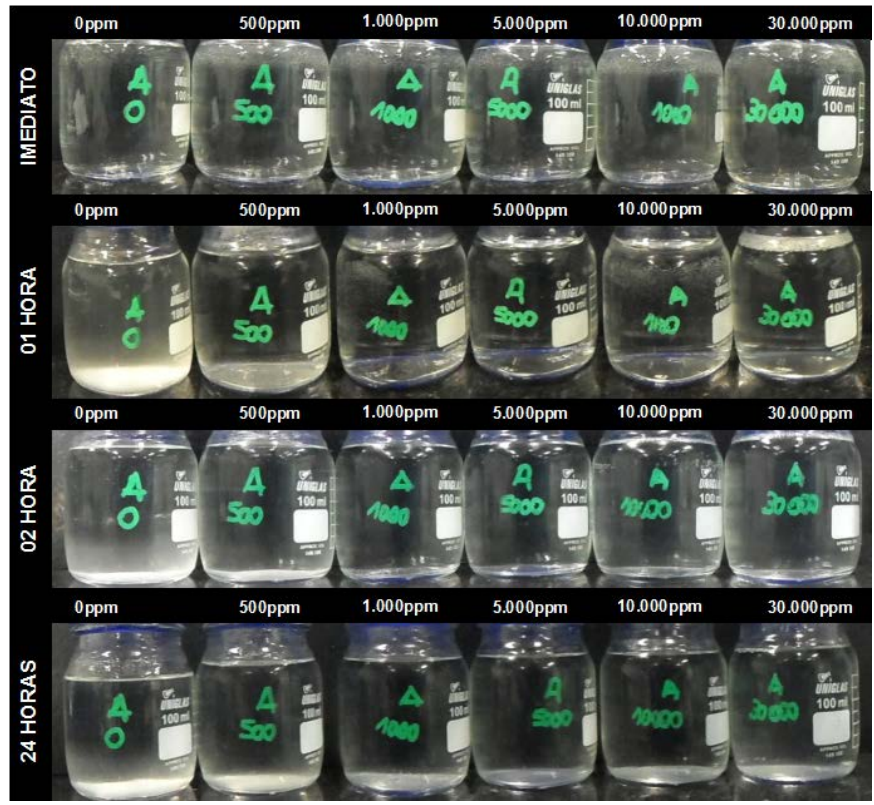

Figura 3: Salmouras na ausência e presença do reagente IN-A em diferentes intervalos de tempo. 


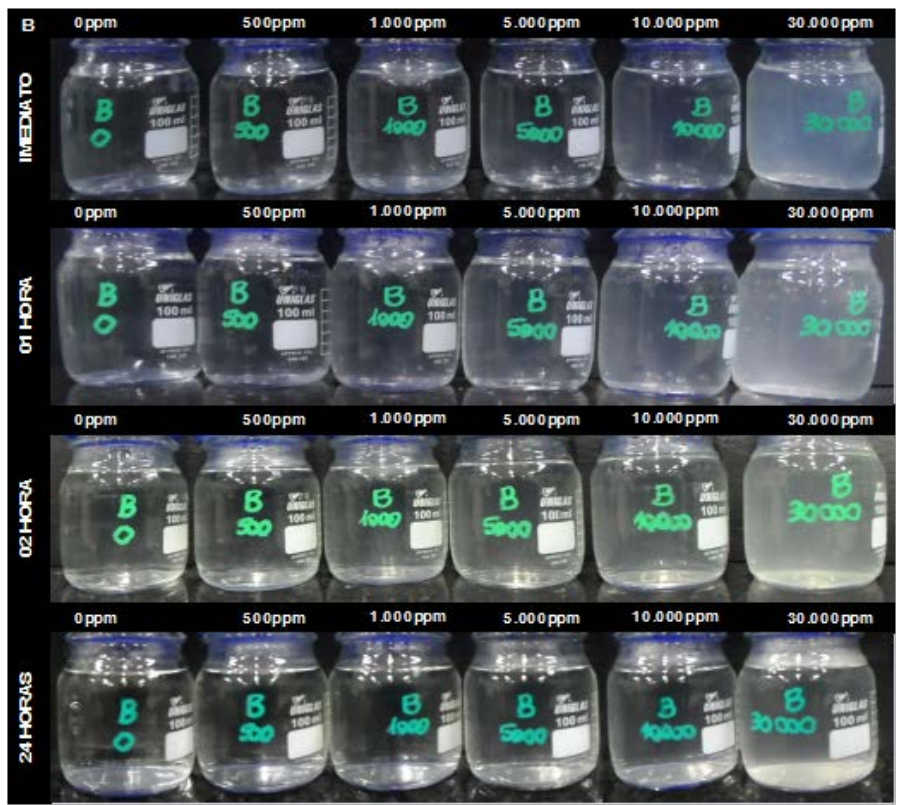

Figura 4: Salmouras na ausência e presença do reagente IN-B em diferentes intervalos de tempo.

\section{CONCLUSÕES}

Os estudos realizados no presente trabalho mostraram as diferentes ações de dois inibidores de incrustação (IN-A e IN-B) formulados e investigados para evitar a incrustação em condições de extração de petróleo em plataformas brasileiras., evidenciando que as características de solubilidade nas condições do processo de extração devem ser consideradas para a obtenção de um reagente de elevadas eficiências e compatibilidades. O IN-A promoveu resultados de turbidez menores que 10 NTU em todas as concentrações investigadas, mostrando uma boa compatibilidade com as salmouras. Já o IN-B, obteve menor apresentou valores de turbidez de 12,7; 15,9 e 31,0 NTU nas concentrações de 5, 10 e 30 g/L, respectivamente, sendo incompatível nestas condições. Estes valores mais elevados de turbidez (acima de 10 NTU) foram evidenciados desde o início dos experimentos, sugerindo uma baixa solubilidade do IN-B, possivelmente, devido à sua estrutura polimérica Quanto à eficiência, o IN-A mostrou resultados de, aproximadamente, 80\%, sendo considerado eficiente para uso no processo de extração. Similarmente, o IN-B foi eficiente em baixas concentrações $(0,5$ e 1,0 g/L) com valores de $80,57 \%$ e $80,40 \%$, respectivamente. No entanto, quando aplicado em altas concentrações (5, 10 e $30 \mathrm{~g} / \mathrm{L}$ ) foi ineficiente, possivelmente, devido à uma redução da solubilidade da estrutura polimérica em altas concentrações. Todos os resultados obtidos serão de grande importância e base para a continuidade de estudos para formulação de novos inibidores, considerando uma diversidade de cenários operacionais, principalmente, com a presença de novos íons em solução para composição das salmouras que simulam diferentes plataformas brasileiras de petróleo.

\section{AGRADECIMENTOS}

Os autores agradecem à empresa DorfKetal ${ }^{\mathrm{TM}}$ pelo apoio nos estudos e fornecimento de amostras e informações sobre os inibidores de incrustação.

\section{BIBLIOGRAFIA}

[1] DEMADIS, K. D. et al., "Industrial water systems: problems, challenges and solutions for the process industries", Desalination, v. 213, n. 1-3, pp. 38-46, 2007.

[2] HAARBERG, T. et al., "Scale Formation in Reservoir and Production Equipment During Oil Recovery: An Equilibrium Model", Society of Petroleum Engineers, v. 7, n. 1, pp. 75-84, 1992.

[3] NANCOLLAS, G. H., REDDY, M. M. "The crystallisation of calcium carbonate. II. Calcite growth mechanism", Journal of colloid and interface science, v. 37, n. 4, pp. 824-830, 1971.

[4] SCHAEWER, T. R., BUENO, A. D., BEDRIKOVETSKY, P. Modelagem e simulação da incrustação por sulfato de bário em representações tridimensionais da rocha reservatório: efeitos sobre a permeabilidade, 
In:Proceedings of the 11th Brazilian Congress of Thermal Sciences and Engineering - ENCIT. Curitiba, Brazil: Brazilian Society of Mechanical Sciences and Engineering 2006.

[5] BUTT, F. H., RAHMAN, F., BADURUTHAMAL, U. "Evaluation of SHMP and advanced scale inhibitors for control of CaSO4, SrSO4, and CaCO3 scales in RO desalination", Desalination, v. 109, n. 3, pp. 323332, 1997.

[6] ALMEIDA, B. P. M. et al. $2^{\circ}$ Trabalho de escoamento multifásico incrustações por sulfatos, Universidade Federal do Espirito Santo, 2013.

[7] GILL, J. S. "A novel inhibitor for scale control in water desalination", Desalination, v. 124, n. 1-3, pp. 43-50, 1999.

[8] REIS, M. I. P. et al. Deposição Mineral em Superfícies: Problemas e Oportunidades na Indústria do Petróleo,. Revista Virtual de Química, v. 3, n. 1, p. 12, 2011.

[9] ARAY, A., DUARTE, L. R. Estudo da formação de incrustações carbonáticas. 2010. 48 Curso de Engenharia de Petróleo, Universidade Federal do Rio de Janeiro, Rio de Janeiro.

[10] MCCABE, W. L., SMITH, J., HARRIOTT, P. Crystallization. In: MCCABE, W. L.,SMITH, J., et al (Ed.). Unit Operations of Chemical Engineering: McGraw Hill Chemical Engineering Series, cap.7, 2004.

[11] RABAIOLI, M. R., LOCKHART, T. P. "Solubility and phase behavior of polyacrylate scale inhibitors", Journal of Petroleum Science and Engineering, v. 15, n. 2-4, pp. 115-126, 1996

[12] KUMAR, T., VISHWANATHAM, S., KUNDU, S. S. "A laboratory study on pteroyl-l-glutamic acid as a scale prevention inhibitor of calcium carbonate in aqueous solution of synthetic produced water", Journal of Petroleum Science and Engineering, v. 71, n. 1-2, pp. 1-7, 2010.

[13] KETRANEA, R. et al. "Efficiency of five scale inhibitors on calcium carbonate precipitation from hard water: Effect of temperature and concentration", Desalination, v. 249, n. 3, pp. 1397-1404, 2009.

[14] GOUVEIA, F. "Tecnologia nacional para extrair petróleo e gás do pré-sal", Conhecimento \& Inovação, v. 6, pp. 30-35, 2010.

[15] ASTM. ASTM D511-03 - Standard test methods for calcium and magnesium in water. INTERNATIONAL, A.: West Conshohocken, PA. 11.01: 9 pp. 2009.

[16] NACE. NACE STANDARD TM0374. Laboratory Screening Tests to Determine the Ability of Scale Inhibitors to Prevent the Precipitation of Calcium Sulfate and Calcium Carbonate from Solution (for Oil and Gas Production Systems): 7 pp. 2007.

[17] APHA. Standard Methods for the Examination of Water and Wastewater, 21, Washington, American Public Health Association, 2005.

[18] HOLMBERG, K. et al. Surfactants and polymers in aqueous solution. Second. John Wiley \& Sons, Ltd., 2002.

[19] LEERMAKERS, F. A. M., BALLAUFF, M., BORISOV, O. V. "Counterion Localization in Solutions of Starlike Polyelectrolytes and Colloidal Polyelectrolyte Brushes: A Self-Consistent Field Theory", Langmuir, v. 24, p. 10026-10034, 2008.

[20] KABO, V. Y., GORODNOV, V. P. "Precipitação de hydrolysed polyacrylamides with salts of alkali earth metals", Vysokomol. soyed, v. AI9, n. 5, pp. 1109-1112, 1976. 